\title{
Análise de QoE para Streaming de Vídeo em Redes Aéreas 5G mmWave de Conectividade Dupla
}

\author{
Ronilson W. S. Pereira ${ }^{1}$, Marcelino S. Silva ${ }^{1}$, José Jailton ${ }^{1}$ \\ Instituto de Tecnologia - Universidade Federal do Pará (UFPA) \\ Caixa Postal 8619 - 66.075.110 - Belém - PA - Brasil \\ ronilsonengenharia@gmail.com, \{marcelino,jjj\}@ufpa.br
}

\begin{abstract}
The millimeter wave communication (mmWave) appears as an essential part for future fifth generation $(5 G)$ networks, as they adopt higher carrier frequencies offering high bandwidth, lower latency, however, the system performance degrades due to the sensitivity of the links to obstacles, so it is necessary to combine the $5 G$ mmWave with UAV. This work presents an analysis of the Quality of Experience (QoE) perceived by the user, in video transmission in mmWave dual communication system using UAV-BS. The results show the efficiency of this system for multimedia applications using videos, improving the QoE of wireless users by $43 \%$.
\end{abstract}

Resumo. A comunicação de ondas milimétricas (mmWave) surge como parte essencial para as futuras redes de quinta geração (5G), pois adotam frequências portadoras mais altas oferecendo alta largura de banda, menor latência, entretanto, o desempenho do sistema se degrada devido à sensibilidade dos links para obstáculos, por isso torna-se necessário a combinação do $5 \mathrm{G}$ mmWave com UAV. Este trabalho apresenta uma análise da Qualidade de Experiência (QoE) percebida pelo usuário, na transmissão de vídeo num sistema mmWave de comunicação dupla utilizando UAV-BS. Os resultados apontam a eficiência desse sistema para aplicações multimídia utilizando vídeos, melhorando a QoE dos usuários sem fio em $43 \%$.

\section{Introdução}

Devido ao avanço nas comunicações e o crescimento do número de dispositivos móveis e diversas aplicações que utilizam streaming de vídeo que necessitam de baixa latência e altas taxas de dados, a demanda pela comunicação móvel sem fio está crescendo rapidamente. $\mathrm{O}$ crescimento do tráfego está sendo impulsionado pelo número crescente de assinaturas de smartphones e pelo aumento do volume médio de dados por assinatura, alimentado principalmente por mais visualização de conteúdo de vídeo. Em 2026, espera-se que as redes $5 \mathrm{G}$ transportem 53 por cento do tráfego total de dados móveis [Ericcson 2020].

O uso de estações base (BSs) no ar utilizando veículos aéreos não tripulados (UAVs) ou rede ad hoc voadora (FANET), é uma tecnologia promissora para cenários de usuários densos fornecendo um complemento importante para redes ultradensas de pequenas células. Uma das aplicações mais importantes é aumentar a cobertura de redes de comunicações móveis [Fotouhi et al. 2021]. 
A proposta apresentada em [Li et al. 2019] estuda um sistema de conectividade dupla UAV-BS em redes sem fio, onde combina a alta mobilidade de veículos aéreos não tripulados e conectividade dupla para fornecer aos usuários taxas de dados e serviços de comunicação onipresentes em cenários de usuários densos. Este trabalho propõe uma arquitetura 5G mmWave de conectividade dupla (CD) utilizando UAV-BS para fornecer garantias de conectividade e qualidade de experiência em streaming de vídeo.

A literatura atual apresenta interessantes estudos sobre a utilização eficiente de recursos da rede com a implementação de UAV-BS, como em [Fotouhi et al. 2021] e [Li et al. 2019]. De forma complementar, a contribuição deste artigo consiste em apresentar um estudo sobre a percepção do usuário em relação a qualidade geral do serviço de streaming de vídeo em sistema sem fío mmWave de comunicação dupla UAV-BS.

\section{Trabalhos Relacionados}

A proposta apresentada em [Su and Maw 2020], realiza uma análise comparativa de streaming de vídeo em rede definida por software concentrando principalmente no redirecionamento do tráfego, abordagem para melhorar as taxas de bits de streaming de vídeo e para reduzir a perda de pacotes no vídeo aprimorado proposto, entretanto, não foi considerada a percepção do usuário na qualidade geral de serviço considerando os fatores de influência mostrados nos resultados.

De acordo com [Salva-Garcia et al. 2018], os autores propuseram uma nova estrutura 5G-UHD para alcançar streaming de vídeo adaptável tendo em vista um grande desafio técnico para atender à crescente expectativa dos usuários de ultra alta definição (UHD) de entrega contínua de vídeo de alta qualidade em pontos de acesso 5G, onde é comum ocorrer congestionamento. Dentre das principais vantagens esperadas nas futuras redes $5 \mathrm{G}$, espera-se lidar com streaming de vídeo UHD.

Outros autores como [Ali Muthanna et al. 2020], ressaltam o crescente interesse em aplicativos baseados em vídeo em que resultou em um rápido aumento no tráfego de dados sem fio e atendendo às rigorosas demandas de qualidade de experiência. $\mathrm{O}$ trabalho apresenta modelos de transmissão de tráfego de vídeo em redes $5 \mathrm{G}$ com base em ondas milimétricas em conjunção com unidades de nuvem no limite de rede de acesso de rádio (RAN) para descarregar a rede subjacente atingindo assim maior eficiência em termos de descarga, sobrecarga, cobertura e atraso.

A proposta apresentada em [Kang et al. 2019], a conectividade múltipla foi proposta para resolver o problema de degradação de desempenho em mmWave devido a aumento do serviço, tempo de inatividade e travamentos, visto que, usuários móveis em uma rede celular ultradensa de ondas milimétricas experimentam eventos de transferência com mais frequência do que em redes convencionais.

\section{Fundamentação Teórica}

\subsection{Quinta Geração de Redes Móveis (5G)}

Com os problemas atuais que a tecnologia $4 \mathrm{G}$ vem enfrentando relacionados a requisitos de desempenho como alta latência, baixa transferências de dados, com a quinta geração 
de redes celulares $5 \mathrm{G}$, espera-se cenários flexíveis, possuindo uma baixa latência de aproximadamente $1 \mathrm{~ms}$, número massivo de conexões e altas taxas de transferências de dados.

A quinta geração de redes móveis $(5 \mathrm{G})$ visa resolver os requisitos substancialmente aumentados de taxa de dados, volume de tráfego e o crescimento maciço de dispositivos conectados. Para atender a este enorme aumento da capacidade de tráfego de dados sem fio, há três métodos: um é melhorar a eficiência espectral, outro é para melhorar a reutilização do espectro, e a última é aumentar a largura de banda. Entre os métodos mencionados, o mais direto e promissor é aumentar a largura de banda para transmissão de dados sem fio. Uma das soluções promissoras é usar a banda de frequência de ondas milimétricas para obter um amplo espectro disponível [Chen 2020].

Com o desenvolvimento intenso da tecnologia $5 \mathrm{G}$ que foi projetada para interconectar não apenas pessoas, mas espera-se que as cidades no futuro tenham um sistema totalmente interligado, onde teremos um grande número de itens inteligentes e componentes de softwares conectados. A Internet das Coisas (IoT) em sistemas sem fio 5G será uma virada de jogo na geração futura, isso abrirá uma porta para uma nova arquitetura sem fio e serviços inteligentes. Esta tecnologia emergente terá um papel vital papel na evolução dos aplicativos de hoje que ainda precisam melhorias [Bhajantri and Gangadharaiah 2020].

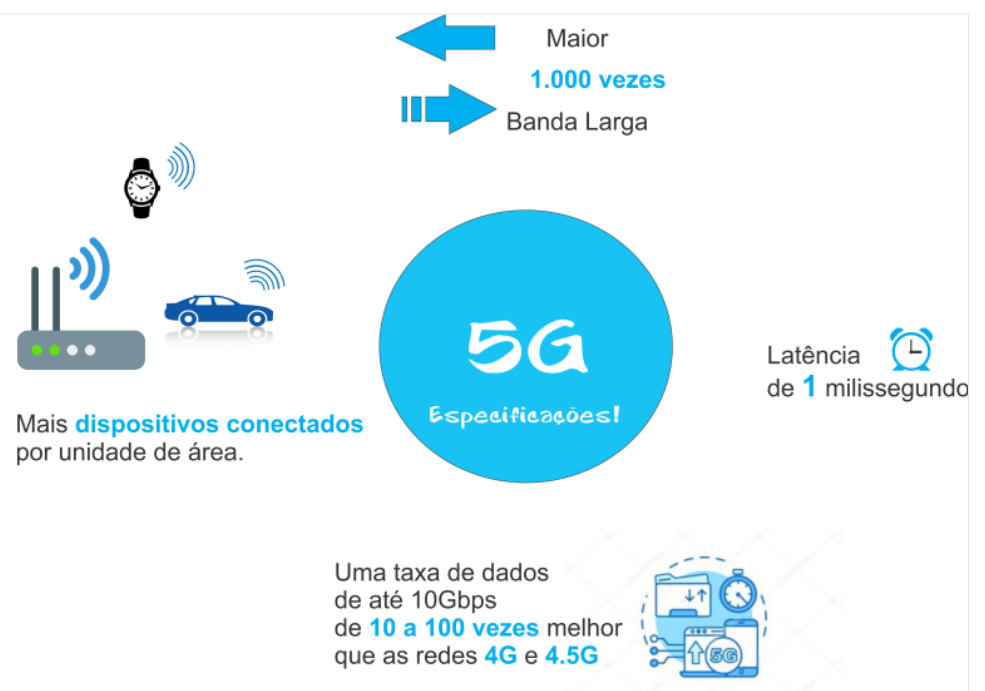

Figura 1. Especificações Tecnologia 5G

As principais pesquisas relacionadas ao sistema 5G ilustradas na Figura 1 focam principalmente nos problemas como a taxa de dados, em que a transferência de dados deve ser 10 vezes mais do que as tecnologias existentes, baixa latência, a latência deve ser 10 vezes menor em comparação com as atuais redes LTE, temos também a alta largura de banda e eficiência de espectro, a tecnologia 5G exigem alta largura de banda, o que pode ser alcançado através do uso de antena MIMO e ondas milimétricas [Shayea et al. 2020]. As comunicações 5G mmWave podem trazer vários benefícios para conectividade sem fio e melhorar o desempenho de redes celulares, além disso, ao associar UAV com mmWave, podemos criar um novo tipo de redes celulares. 


\subsection{Millimeter Waves}

Atualmente, aplicações intensivas de largura de banda emergentes estão presentes nas rotinas diárias de usuários móveis. Com a sobrecarga do espectro LTE na faixa de frequência abaixo de $6 \mathrm{GHz}$ surge com as redes $5 \mathrm{G}$ o interesse de se utilizar bandas mmWaves que são caracterizadas por seus pequenos comprimentos de ondas, alta frequência e grande largura de banda.

Em relação ao uso de bandas acima de $6 \mathrm{GHz}$ para comunicação, tem havido pesquisas substanciais nos últimos anos em relação ao potencial de comunicação celular em bandas de ondas milimétricas que abrangem o intervalo entre 30 e $100 \mathrm{GHz}$. Essas frequências estão sujeitas a uma maior perda de percurso em comparação com as bandas sub-6 GHz usadas atualmente [Ntontin et al. 2020]. A maior disponibilidade de espectro desocupadas em frequências mmWave é uma das principais vantagens das comunicações 5G mmWave, a largura de banda maior se traduz em taxas de dados extremamente altas, atingindo rapidamente as taxas de pico de dados de $10 \mathrm{Gbit} / \mathrm{s}$ [Parida et al. 2020].

O uso de ondas milimétricas possui suas vantagens técnicas e desafios, seu uso para serviços de celular se tornou uma realidade. Seu desempenho eficiente para comunicações móveis, por exemplo, alta largura de banda, velocidades super-rápidas, latência ultrabaixa e maior conectividade. Entretanto, o efeito de bloqueio é um dos principais desafios técnicos, os sinais mmWave são bloqueados por obstáculos físicos em seus caminhos de propagação, sinais mmWave também são afetados por atenuação atmosférica restringindo sua faixa de transmissão no espaço livre.

\section{Materiais e Métodos}

Os experimentos foram conduzidos sobre simulações criadas utilizando o Network Simulator 3 (NS3) usando o módulo mmWave que é capaz de realizar simulações com dispositivos móveis (UEs) conectados a um eNB LTE e para um eNB mmWave [Mezzavilla et al. 2018]. Consideramos dois cenários neste estudo, onde temos a conectividade dupla utilizando ondas milimétricas habilitadas para UAV e a rede LTE, durante a simulação o UAV-BS está conectado ao UE por links mmWave que vai ter transmissão multimídia. Simulamos um cenário tradicional utilizando apenas a interface 4G para fazer um comparativo da qualidade de experiência.

Para fazer a avaliação da QoE com relação ao serviço de vídeo, utilizou-se métricas de avaliação objetivas que são baseadas em modelos matemáticos para estimar a média da opinião dos usuários, calculando o grau de distorção das sequências de saída em relação às sequências de entrada. As métricas consideradas foram SSIM (Structural Similarity Index) e VQM (Video Quality Metric), visto que, ambas têm uma boa correlação com o feedback subjetivo dos usuários, ambos os cenários possuem aplicação do tipo vídeo (streaming) no formato 4K sobre os dispositivos móveis.

A métrica SSIM estima a similaridade entre o vídeo original e o vídeo degradado comparando parâmetros como luminosidade, contraste e sua estrutura, seus valores variam no intervalo de 0 a 1 , quanto maior for a média melhor será sua visualização. $\mathrm{O}$ VQM calcula uma métrica de qualidade geral considerando alguns aspectos como ruído, distorção dos frames, sua escala de resultado varia entre 0 a 5 sendo 0 o melhor valor possível [Van Der Hooft et al. 2020]. 


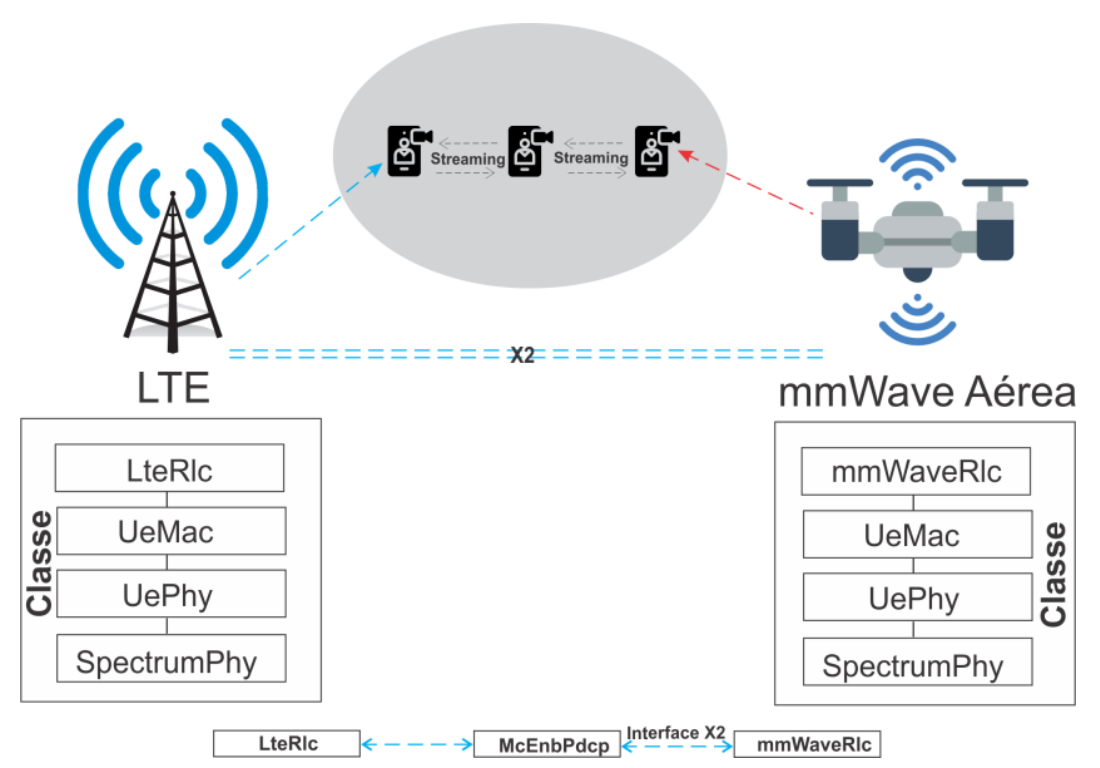

Figura 2. Arquitetura de Conectividade Dupla UAV- BS

A Figura 2 apresenta o diagrama da arquitetura proposta onde, sistemas mmWave enfrentam muitos problemas como alta variabilidade do canal, bloqueio humano e provavelmente usarão a conexão dupla, neste cenário o UAV-BS está fornecendo cobertura aos usuários através de links mmWave com objetivo de maximizar a área. Assumimos que todos os UAVs têm energia suficiente para chegar à sua posição 3D no ar para realizar a comunicação com os UEs com altitude típica de 10 metros.

Analisamos a arquitetura que consiste no eNB LTE, no UAV-BS mmWave e nos UEs utilizando aplicação do tipo vídeo (streaming) (Fig. 2). O módulo de comunicação dupla do NS3 assume que as redes centrais do LTE e do mmWave são integrados, sendo assim, os eNBs do LTE e o mmWave compartilham a mesma rede de backhaul, isto é, ambos estão conectados uns aos outros através de links X2. Para realiza a integração utilizamos a camada PDCP (Packet Data Convergence Protocol), uma vez que permite uma implantação não localizada dos eNBs e uma abordagem limpa na concepção das camadas Phy, Mac e Rlc. O modelo de canal usado para as simulações neste trabalho é baseado no modelo de canal 3GPP para frequências de $6-100 \mathrm{GHz}$ descrito em [Mezzavilla et al. 2018].

\section{Resultados}

Os resultados das métricas SSIM e VQM foram obtidos executando 10 simulações e calculando a média com o objetivo de avaliar o sinal de vídeo degradado em relação ao de referência. No primeiro cenário utilizando a interface com conectividade dupla, criamos um contêiner com 20 UEs que estão se movendo de acordo com o modelo de mobilidade (Random Waypoint) [Althunibat et al. 2019] próximo de duas BS LTE localizadas no mesmo local e duas UAV-BS. O segundo cenário em vez de usar conectividade dupla temos apenas a interface LTE, os parâmetros de simulação são apresentados na Tabela 1. 
Tabela 1. Parâmetros Gerais da Simulação

\begin{tabular}{|c|c|}
\hline Parâmetros & Valor \\
\hline Número de UEs & 20 \\
\hline Qtd. Estação Base (BS) & 2 (LTE) / 2 (UAV mmWave) \\
\hline Área de Cobertura (km2) & 4 \\
\hline Potência de Transmissão (dBm) & 40 (LTE) / 20 (UAV mmWave) \\
\hline Frequências (GHz) & $2.6(\mathrm{LTE}) / 26(\mathrm{mmWave})$ \\
\hline Tempo de Simulação & $250 \mathrm{~s}$ \\
\hline
\end{tabular}

O fluxo dos vídeos foi ponta a ponta e o cenário foi controlado, isto é, sem nenhum tráfego de dados adicional que pudesse influenciar nas avaliações realizadas (os demais dispositivos não são consumidores). Conforme a Figura 3, a média geral SSIM para o cenário utilizando a interface com conectividade dupla foi de 0,98 mostrando uma alta qualidade de transmissão. Já no cenário utilizando apenas o LTE o vídeo recebido pelo dispositivo obteve média SSIM igual a 0,55 mostrando uma alta degradação na qualidade do vídeo.

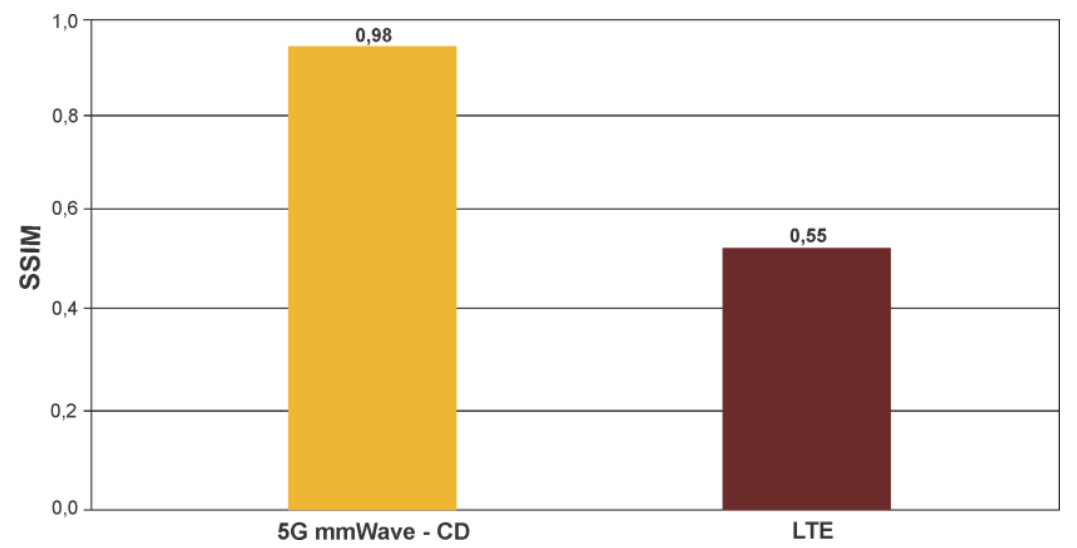

Figura 3. Média SSIM

Com base nos resultados da Tabela 2 e no gráfico da Figura 3, fica claro que a média geral do SSIM do vídeo $4 \mathrm{~K}$ simulado no sistema proposto é próximo a 1 , sendo que valores altos são melhores para a métrica, o que significa uma correlação perfeita de duas imagens semelhantes.

Tabela 2. Valores da Métrica SSIM

\begin{tabular}{|c|c|c|}
\hline Valores & SSIM - 5G mmWave (DC) & SSIM - LTE \\
\hline Valor Mín. & 0,991059 & 0,524945 \\
\hline Valor Máx. & 0,988992 & 0,558369 \\
\hline Valor Médio & 0,988596 & 0,557932 \\
\hline
\end{tabular}

Considerando a métrica VQM, os vídeos que registram valores próximos de zero são considerados de boa qualidade e valores afastados de zero demostram uma qualidade de transmissão ruim. De acordo com os dados apresentados na Tabela 3, os 
valores mínimos da métrica no sistema proposto e usando a interface LTE obtiveram 0,49 e 3,92 de VQM respectivamente.

Tabela 3. Valores da Métrica VQM

\begin{tabular}{|c|c|c|}
\hline Valores & VQM - 5G mmWave (CD) & VQM - LTE \\
\hline Valor Mín. & 0,498391 & 3,924853 \\
\hline Valor Máx. & 0,537418 & 4,372938 \\
\hline Valor Médio & 0,499727 & 4,339472 \\
\hline
\end{tabular}

A Figura 4 apresenta os resultados da métrica VQM, em que identificamos valores próximos de 0 no cenário $5 \mathrm{G}$ mmWave - CD obtendo 0,49 de VQM demostram uma ótima qualidade de transmissão. A transmissão no cenário LTE o vídeo obteve média 4,33 de VQM mostrando maior perda de qualidade e confirmando o quanto foi ruim a qualidade de transmissão do vídeo.

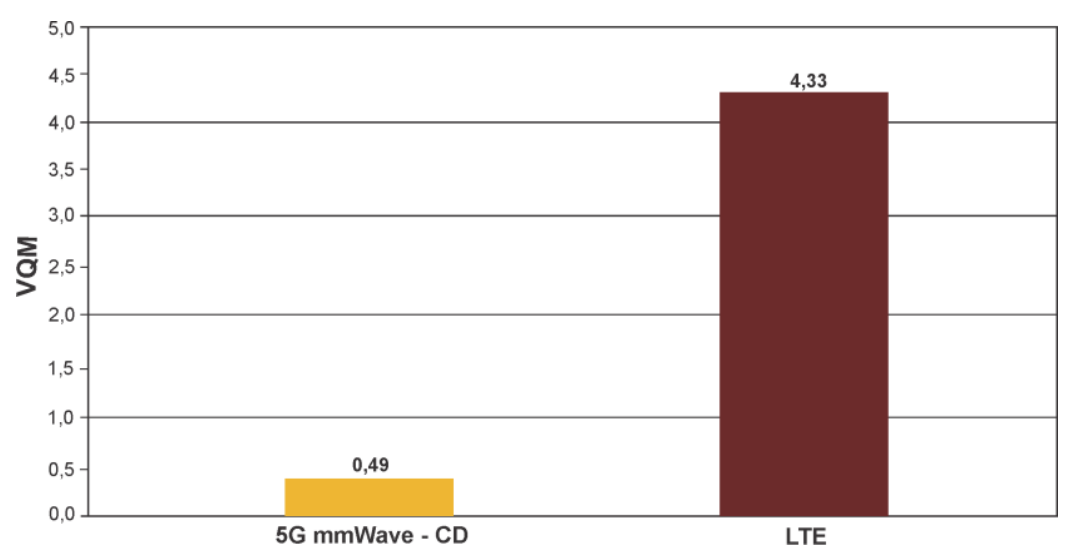

Figura 4. Média VQM

\section{Conclusão}

Os resultados mostraram que a transmissão do serviço de streaming de vídeo no sistema mmWave de comunicação dupla UAV-BS é mais eficaz melhorando a QoE em $43 \% \mathrm{em}$ comparação a utilização apenas da interface LTE. Os benefícios que redes $5 \mathrm{G}$ mmWave irão proporcionar para aplicações multimídia utilizando vídeos como qualidade na transmissão, entrega confiável, menor distorção dos quadros permitindo uma melhor percepção do usuário, os resultados deste estudo apontam a viabilidade de se utilizar o sistema. Como trabalhos futuros, pretende-se verificar a qualidade de acordo com a mobilidade do usuário e conforme o mesmo vai se conectando em várias BS (avaliar o handover).

\section{Referências}

Ali Muthanna, M. S., Mohammed Obadi Musaeed, A., Muthanna, A., Filimonova, M. and Hazzaa Esmail, Y. A. (2020). Analysis of the Advantages of Millimeter Waves for Video Traffic Transmission in 5G Networks. Proceedings of the 2020 IEEE Conference of Russian Young Researchers in Electrical and Electronic Engineering, EIConRus 2020, p. 51-53. 
Althunibat, S., Badarneh, O. S. and Mesleh, R. (2019). Random Waypoint Mobility Model in Space Modulation Systems. IEEE Communications Letters, v. 23, n. 5, p. 884-887.

Bhajantri, L. B. and Gangadharaiah, S. (2020). A comprehensive survey on resource management in internet of things. Journal of Telecommunications and Information Technology, v. 2020, n. 4, p. 27-43.

Chen, W. C. (2020). 5G mmWAVE Technology Design Challenges and Development Trends. 2020 International Symposium on VLSI Design, Automation and Test, VLSIDAT 2020, p. 5-8.

Ericcson (2020). Ericsson Mobility Report - November 2020. Ericsson, n. November, p. 36.

Fotouhi, A., Ding, M. and Hassan, M. (2021). DroneCells: Improving spectral efficiency using drone-mounted flying base stations. Journal of Network and Computer Applications, v. 174, p. 1-14.

Kang, S., Choi, S., Lee, G. and Bahk, S. (2019). A dual-connection based handover scheme for ultra-dense millimeter-wave cellular networks. 2019 IEEE Global Communications Conference, GLOBECOM 2019 - Proceedings, p. 0-5.

Li, Z., Wang, Y. and Liu, Y. (2019). Efficient Resource Allocation in UAV-BS Dual Connectivity Heterogeneous Networks. 9th International Conference on Information Science and Technology, ICIST 2019, p. 234-239.

Mezzavilla, M., Zhang, M., Polese, M., et al. (2018). End-to-end simulation of 5G mmWave networks. IEEE Communications Surveys and Tutorials, v. 20, n. 3, p. 2237-2263.

Ntontin, K., Verikoukis, C. and Member, S. (2020). Relay-Aided Outdoor-to-Indoor Communication in Millimeter-Wave Cellular Networks. v. 14, n. 2, p. 2473-2484.

Parida, S., Majhi, S. and Das, S. K. (2020). Wireless Powered Microwave and mmWave based Communication Networks - A Survey. Proceedings of the 5th International Conference on Inventive Computation Technologies, ICICT 2020, p. 98-102.

Salva-Garcia, P., Alcaraz-Calero, J. M., Alaez, R. M., et al. (2018). 5G-UHD: Design, prototyping and empirical evaluation of adaptive Ultra-High-Definition video streaming based on scalable H.265 in virtualised 5G networks. Computer Communications, v. 118, n. April 2017, p. 171-184.

Shayea, I., Ergen, M., Azmi, M. H., et al. (2020). Key challenges, drivers and solutions for mobility management in 5G networks: A survey. IEEE Access, v. 8, p. 172534172552 .

Su, H. M. and Maw, A. H. (2020). Comparative Analysis of Video Streaming in Software Defined Networking. Proceedings of the 4th International Conference on Advanced Information Technologies, ICAIT 2020, p. 12-17.

Van Der Hooft, J., Vega, M. T., Timmerer, C., et al. (2020). Objective and Subjective QoE Evaluation for Adaptive Point Cloud Streaming. 2020 12th International Conference on Quality of Multimedia Experience, QoMEX 2020, p. 1-6. 